\section{New legislation forecast}

THE British government has taken a further step towards tighter legislation on animal experiments in a second White Paper (policy document) on Scientific procedures on living animals, published last week by the Home Office, the government department responsible (Cmnd 9521, HMSO, £3.30). The document is said to be the result of collaboration between the government, scientists and the "realistic element of the animal welfare movement". The main changes from the earlier proposals (see Nature 303, 191; 1983) are: tighter controls on the severity of procedures; modification of the project licensing assessment system; improvements in laboratory animal care; higher penalties for some offences.

Under the new proposals, investigators will need two separate licences, both a personal licence betokening general competence and a project licence for each proposed investigation. Applicants for both kinds of licence must provide the Home Secretary with detailed information about themselves and their proposed work, together with the names of persons willing to act as sponsors. For a personal licence, the sponsor will vouch for the applicant's "competence and integrity". For project licences, the sponsor will give a detailed opinion of the scientific merits of the project and techniques to be used. The white paper says that a field trial has shown that such a system would work in practice. But because of the practical difficulties of assessing all project licence applications in this way, the Home Office now proposed to refer only those projects where the scientific quality of the work is in doubt to an independent assessor.

Criteria for obtaining a licence under the proposed regime will be stricter than at present. The Home Secretary must be persuaded that the work is justifiable, that no satisfactory alternative to the use of animals is available, that the minimum number of animals is used and that the least possible suffering is caused.

Considerable emphasis is placed on the severity of pain, which will be balanced against potential benefit when project licence applications are considered. Such a cost-benefit principle has been widely advocated, in particular by Sir Andrew Huxley, president of the Royal Society, who suggested a graded approach to the justification of experiments on animals in his anniversary address to the Royal Society last year.

The new white paper is an excellent compromise in the debate on animal experimentation, Sir Andrew said this week. The licensing system will make clear who is responsible for a project, and the form of permission for project licences will ensure that granting a licence does not become a "rubber stamp procedure".
In practice, the Home Office will issue personal licences which will, in effect, be a certificate of competence to a particular investigator to pursue a particular project. But project licences will be issued only to applicants "who can satisfy the criterion of permissible purpose". The Home Secretary will impose conditions setting " "permissible degrees of severity (of pain) for that project... mild, moderate and substantial". Home Office inspectors will have increased power to order humane killings, "action to be taken at the onset of various signs" such as loss of motor function. The new proposals also require painless killing at the end of a predetermined period, administration of analgesic and constant supervision throughout the procedure.

Before a project licence will be granted, the applicant must agree with the Home Secretary on the likely level of severity of pain an notify an inspector if this forecast is likely to be exceeded. The Home Secretary will decide how the categories of pain are to be defined and applied, and will publish guidelines to inform applicants, licensees and the general public of how the proposals are intended to work.

An animal procedures committee is being set up to advise the government on policy, on applications for research causing "special concern" and on the testing of cosmetics. The committee is chaired by Baroness Warnock and its members include scientists, representatives of organizations that protect animals and representatives of pharmaceutical companies.

The composition of the committee, however, emphasizes the government's commitment to regulated experiments on animals. As Sir Andrew pointed out, the situation cannot arise where experiments can be stopped by a block vote of antiexperimentalists on the committee, which answers the genuine worries of many biological and medical scientists.

Reactions to the white paper are mixed. The Association of British Pharmaceutical Industries accepts the need to update the existing (1876) legislation, but is concerned that there will be "too narrow a definition of the scope of the project licensing provisions". The Humane Research Trust and the British Veterinary Association welcome the proposals in the main. The British Union for the Abolition of Vivisection calls them a "confidence trick" and a "vivisectionists' charter".

The number of experiments on animals in the United Kingdom has fallen from 5.6 million in 1971 to 3.6 million in 1983. In 1985 the Humane Research Trust will give grants of $£ 100,000$ to nineteen investigators using alternative experimental procedures that do not animals, and the pharmaceutical industries will spend $£ 10-12$ million on development of non-animal techniques in drug testing.

Maxine Clarke
US laboratory animals

\section{NIH watchdog committees}

\section{Washington}

LABORATORY animal research at publicly supported institutions in the United States will from November this year have to be overseen by special "animal use" committees that will include at least one lay representative, according to new guidelines issued by the Department of Health and Human Services. The guidelines charge the animal use committees with responsibility for reviewing research programmes and inspecting laboratories and animal houses at least annually. Research that does not meet the committees' standards can be stopped.

The requirement that each committee should include at least one lay member is welcomed by animal welfare campaigners, but some had hoped for a representative concerned specifically with welfare. Welfare champions are unhappy that the committees are not required to review in detail research protocols for investigations in which non-trivial pain may be caused.

Christine Stephens, president of the Animal Welfare Institute, says that this omission is a "profound disappointment". Earlier proposals had required special approval by the committee for non-routine or harmful invasive procedures, procedures involving prolonged restraint, diseases maintained for long periods or nonstandard methods of euthanasia. The new proposals leave it to committee members to decide if special attention is warranted. Ms Stephens is also disturbed that institutions can apparently apply for a retroactive waiver that would release them from their responsibilities under the new guidelines.

The Association of American Universities, in contrast, is relieved that lay members will not be required to review research protocols, a requirement that the association considers "unacceptable".

The guidelines do not have the force of law, although an institution could in theory lose its financial support if deficiencies went uncorrected for long enough. Critics point out, however, that such an occurrence is almost unknown. The National Institutes of Health are still investigating allegations of mistreatment of baboons used in head injury experiments at the University of Pennsylvania; despite the widespread availability of a video tape showing apparent mistreatment, which was last week being shown to members of Congress, the research is still being supported. A bill introduced in the last Congress by Senator Robert Dole and Representative George Brown would have given statutory force to animal use committees: a new version of the bill is likely to be introduced in Congress within the next few weeks.
Tim Beardslev 